\title{
Estimating the Probability of Mining-Induced Seismic Events Using Mine-Scale, Inelastic Numerical Models
}

\author{
D. Beck Beck Arndt Engineering (Pty) Ltd, Australia \\ F. Reusch Beck Arndt Engineering (Pty) Ltd, Australia \\ S. Arndt Beck Arndt Engineering (Pty) Ltd, Australia
}

\begin{abstract}
Detailed, mine-scale non-linear numerical models have been calibrated using observations of displacement and damage at three deep mines. The calibrated models were then used to investigate the correlation between Dissipated Plastic Energy (DPE) and seismic event probability. A strong, non-linear relation between DPE and event probability was observed that describes the development, peak, and decline in seismicity as rock is deformed. The correlations are present for events at all magnitudes, and the where sufficient data was available for a comparison, a relation between DPE and event probability consistent with the relation between event magnitude and frequency described by the Gutenberg Richter equation was found. The application of DPE analysis during the design and sequencing of mines will allow a more quantitative description of seismic hazards than is currently available.
\end{abstract}

\section{Introduction}

In recent times computer and analytical advances have allowed non-linear Finite Element (FE) simulation of the entire volume and excavation history of complex mines. The wide range of valid length scales simulated in these models and the use of higher order elements allows simulation of more complex material behaviour, providing a better numerical platform for testing theories about the causes and effects of induced seismicity. The modern FE models have overcome many of the boundary condition problems, stress-path issues and displacement incompatibility issues common in legacy geotechnical simulation packages.

As the modern, inelastic FE models with higher-order elements produce more realistic estimates of the extent and magnitude of damage in the rock and displacements are more realistic, energy terms are also more accurate, allowing direct comparison of seismic event potential with energy released due to damage simulated in the model.

\section{Model package}

The modelling packages used for this work were ABAQUS Explicit and Implicit. ABAQUS is a specialistoriented, general purpose, 3-D, non-linear, FE, continuum and discontinuum analysis product. It is designed specifically for advanced analysis of problems where there is significant plasticity, high levels of deformation and large numbers of material discontinuities.

\subsection{Material assumptions}

Mohr-Coulomb and Drucker-Prager yield criteria were used to best match the yield criteria developed in previous analyses at the mine. The constitutive model in both cases is a strain softening, dilatant material model. The faults can be modelled either as zones of fault-like material within the continuum, or as contact discontinuities. Contact discontinuities enable the blocks formed by faults to behave in a similar manner to Discrete Elements, except the formulation used is based exclusively on higher order elements and softening and weakening of the faults is described as a function of strain. An advantage of the finely discretised, higher order model elements is that yield through the intact masses formed by faults is very realistic.

An example of fault dislocation and yield through a modelled rock mass is shown for a small volume of rock from the actual model in Figure 1. The volume shown is just a very small portion of a whole model, but 
shows the complex rock phenomena that are simulated and the extent to which the modelled rock may distort. This coupling of discrete movements and intense localised damage of the continuum is essential in some applications to capture important rock mass deformation relevant to the problem.

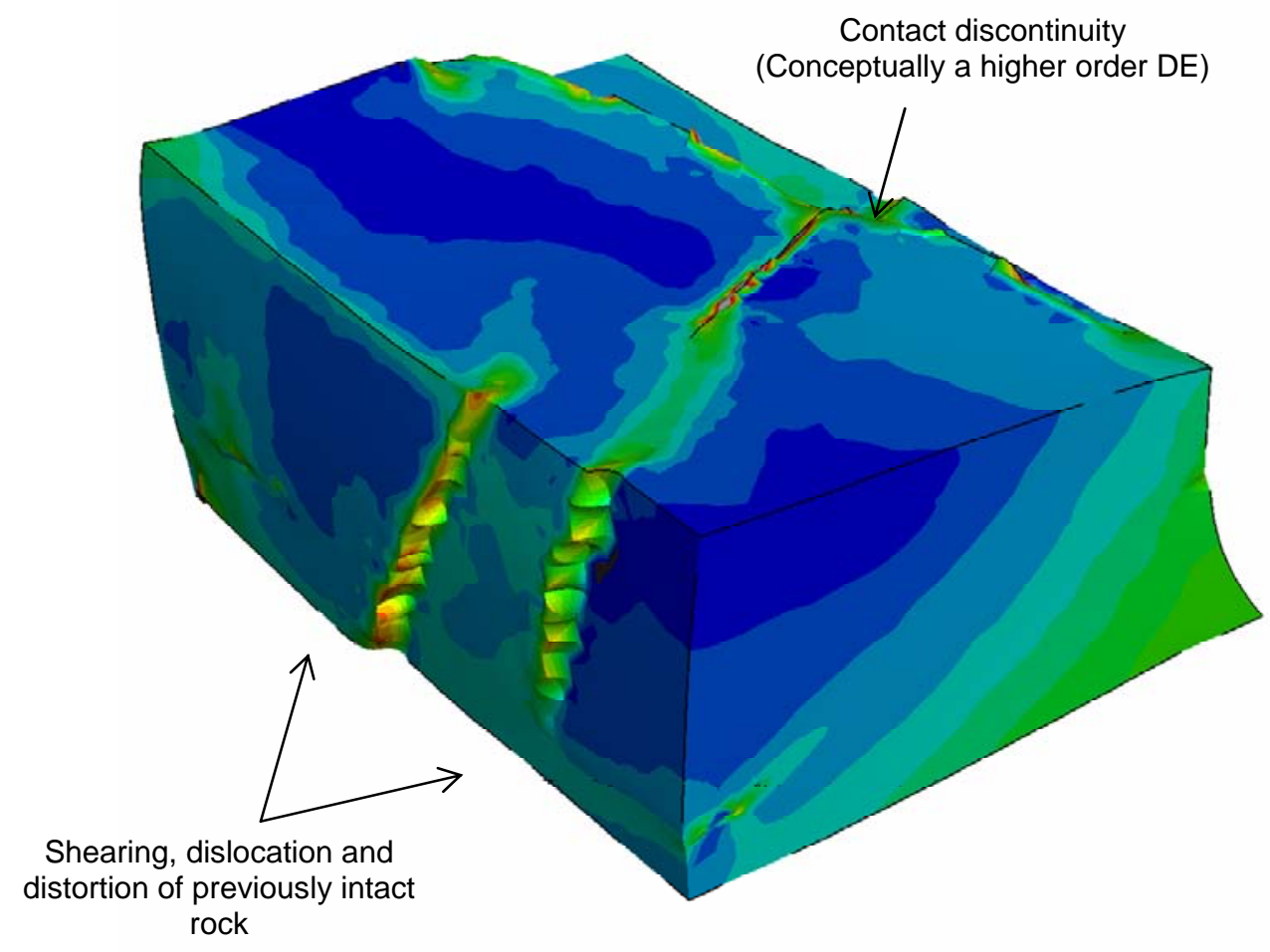

Figure 1 Plastic strain and movement inside a small volume of rock in the model. The dislocation of contact discontinuities at a fault is clear, as is yield, shearing and dislocation through the mass

The inelastic constitutive model for materials and faults in the model assumed that each material has peak and residual properties and that yield results in dilation. Once the peak strength defined by the yield criterion is exceeded, residual properties are introduced. Cohesion, friction angle, stiffness and compressive strength are all reduced as a result of yield and a dilation angle for yielded materials is calibrated. With additional data for calibration, the option exists to extend this behaviour of the continuum parts of the model to incorporate the effects of confinement on residual rock mass behaviour. At this time, high confinement response is most relevant and is what has been calibrated in each case of the case studies, but incorporating more complex, confinement dependent residual response will improve the model accuracy and precision.

The capacity also exists with minimal effort to incorporate additional anisotropic effects due to small scale structures. These small structures could be built discretely as a Discrete Fracture Network (DFN), with the available, mapped fault and joint surfaces represented in a sub model scale, or the capacity exists to incorporate yield on discontinuities at a sub-element scale (nodal) and through the rock mass. If this latter option of a continuum model for joints is used, yield is still simulated on discontinuities and through the mass. This capability is somewhat unique.

\section{Seismogenic correlation}

The seismogenic calibration was described in detail in Beck et al. (2006). In summary, Dissipated Plastic Energy (DPE) is compared to seismic event occurrence using the 'cell evaluation method' described by Beck and Brady (2001), involving discretising the entire model into regular, volumetric 'cells' or 'test blocks'.

DPE release rate is calculated in each of the test blocks for historic mining steps and compared to the proportion of blocks for each range of DPE release rate that contain and do not contain events. The relation 
between DPE release rate and the event probability, $(x)$ of a mine tremor of a certain magnitude, $X$, occurring in a test block, is denoted:

$$
p(X)=x \approx \frac{n_{D P E i}}{e_{D P E i_{j}}}
$$

Where the total number of test blocks having values within any range of DPE release rate is denoted $e_{D P E_{i}}$ where $i$ is the fixed interval of DPE being evaluated (e.g. $1000 \mathrm{~J} / \mathrm{m}^{3}$ to $2000 \mathrm{~J} / \mathrm{m}^{3}$ ) and the sum of blocks containing events within that magnitude range as $n_{D P E j}$, where $j$ is the event magnitude range (e.g. $0 \mathrm{M}_{\mathrm{L}}$ to $1 \mathrm{M}_{\mathrm{L}}$ ) being considered.

\subsection{Mine 1: Continuous advance sequence, 1 year study}

Mine 1 is discussed in detail in Beck et; al., (2006). Mine 1 is an example of seismogenic calibration of a model where a moderate amount of data is available. By world standards, the extent of the seismic array and the sensor density are only moderate, typical for large Australian underground mines.

The model of the mine was first calibrated using damage, displacement and ground support performance data. The seismogenic correlation procedure was then performed using 12 months of data, simulated in monthly excavation steps. Larger model steps were used in excavation steps preceding the study period, but in all over 50 model steps were required to re-create a realistic stress path.

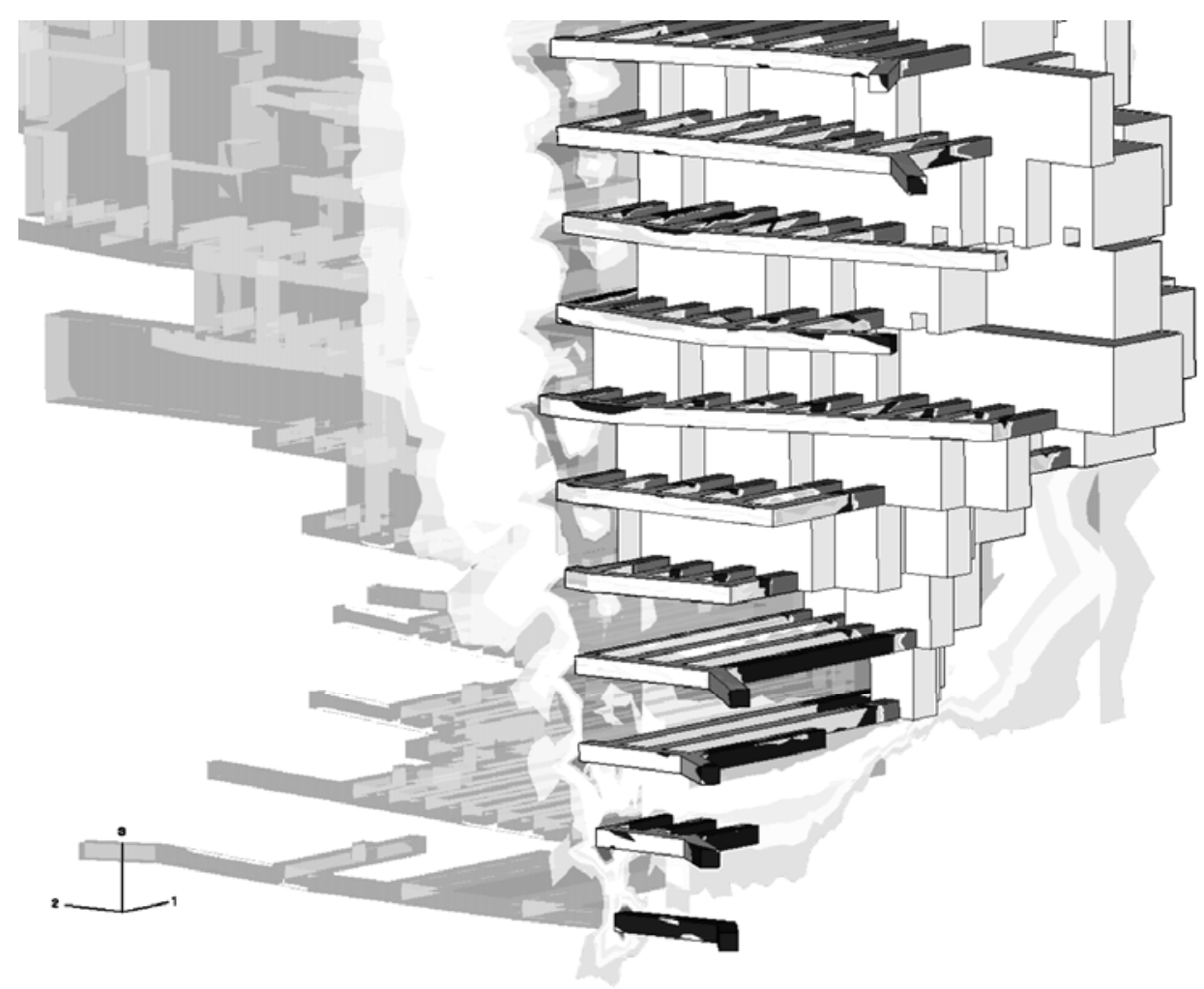

Figure 2 Example of the FE model complexity used in the study at Mine 1

An example of the FE model complexity is shown in Figure 2. This step in the model shows plastic strain on a section through the rock mass, as well as support load contoured on drive surfaces.

The results of the DPE calibration (Figure 3) show that the boundary for event occurrence is continuous and bounded with a near-zero event probability at zero DPE release rate. Essentially, this means there are almost no seismic events not accounted for by the DPE relation. 
The figure also shows that there is a peak DPE beyond which the event probability decreases. This occurs because beyond this limit, the ground has been conditioned (softened by damage) and seismic activity must therefore decrease. For forecasting purposes, it is assumed that, for this particular mine, once a total DPE (cumulative for all steps) exceeds $5000 \mathrm{~J} / \mathrm{m}^{3}$ in previous mining steps, no more seismicity should be expected, and this has proven to be a reliable limit. If greater than $2000 \mathrm{~J} / \mathrm{m}^{3}$ DPE is released in a test block in a single step, the probability decreases as shown in the graph. Smaller mining steps and smaller prediction periods would reduce the number of test cells affected by excessive deformation during an individual mining step.
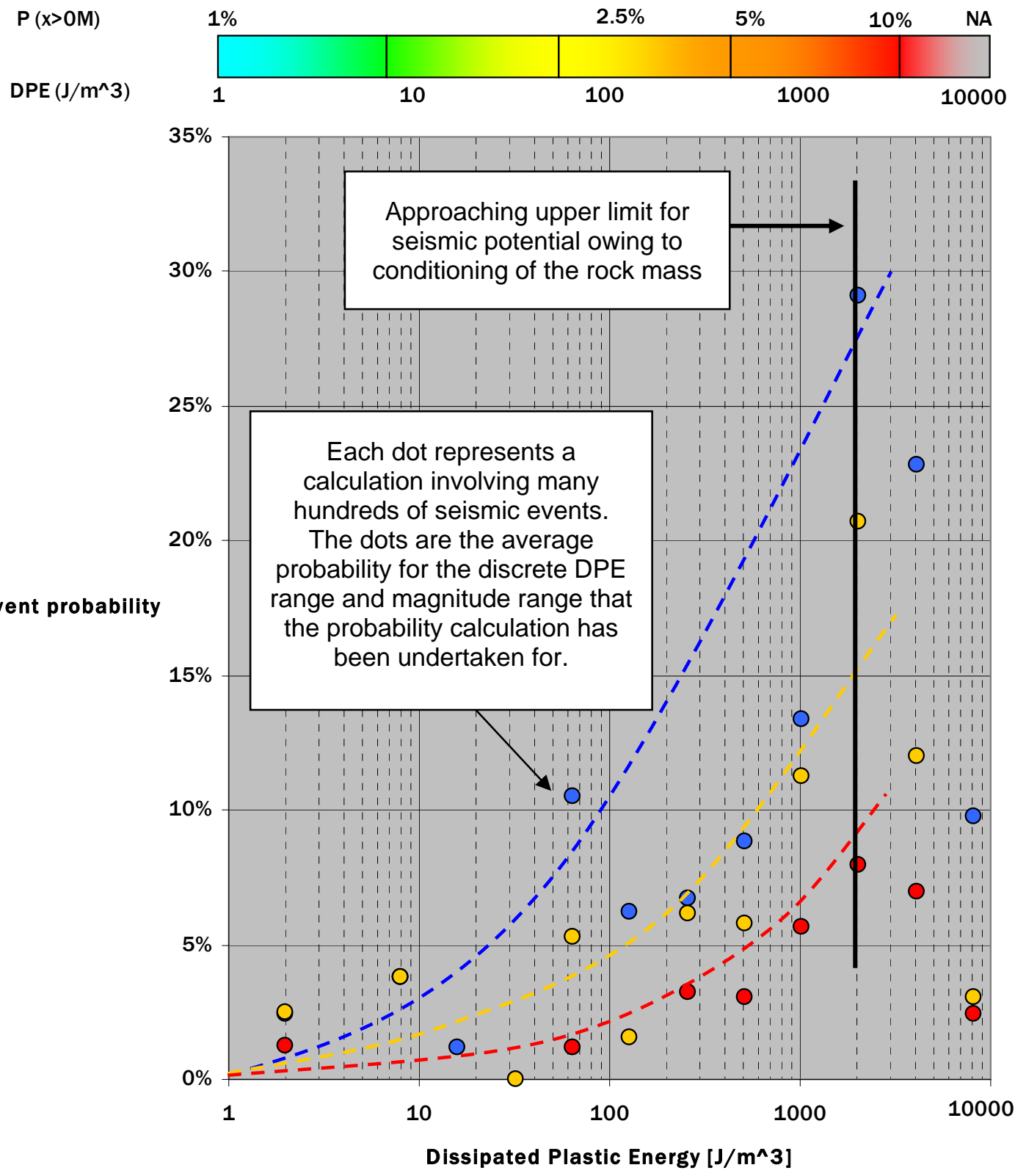

Any Event $0>0 \mathrm{M}$ O >-1.0M

Figure 3 Correlation between event probability and Dissipated Plastic Energy [DPE] for a 1 month modelling step 
There is a large amount of information in the graph shown in Figure 3:

1. The graph allows the modelled DPE to be interpreted as event probability directly with a quantifiable error range.

2. The 'mean efficacy' for each magnitude shown in the graph is the average correlation between event occurrence and DPE, expressed as a probability over the period of the study period. The mean efficacy is the relation that would normally be used to quantify the relation between DPE and event probability.

3. The graphs show there is near-zero event probability at zero DPE release rate. Essentially, this means there are relatively few unaccounted for seismic events (seismic events which occur where there is no DPE). This implies a good model calibration, because the limits of the seismogenic zone (the zero event probability contour) should correspond to the zero DPE contour, as the zero DPE contour in the model is where the model indicates no new rock mass damage is occurring.

4. The figures also show that there is a peak DPE beyond which the event probability begins to decrease. This occurs because beyond this limit, the ground has been conditioned (softened by damage) and seismic activity must therefore decrease.

\subsection{Mine 2: Remnant mining, 7 year study}

The same calibration procedure was performed at Mine 2 for all seismic events occurring within $250 \mathrm{~m}$ of a location of interest for seven years of data and the results are presented in Figures 3 to 5 .

This study differs from mine 1 owing to the very large number of seismic events, the extensive time span of the data as well as the extent of mining that was undertaken during the study period. It also differs because Mine 1 employed a continuously advancing mining sequence, but Mine 2 is almost exclusively a remnant mine. This is relevant because the typical stress path differs considerably in each case.

Again, the mine model was calibrated using displacements before the seismogenic correlation was tested. As for the Mine 1 calculation, each visible data point represents a calculation involving many hundreds of seismic events within a certain magnitude and DPE range. The data points are the average probability (not single events) for a discrete DPE range that the probability calculation has been undertaken for. In this case the probability equates to the chance of having an event at the indicated magnitude, within $20 \mathrm{~m}$ of the test location during the time period of the model step.

Similar conclusions can be drawn from the Mine 2 relations as were drawn from the Mine 1 study, but in this case, a difference between the long term 'mean efficacy' and shorter periods was found. This can be seen in Figure 4, which shows the mean correlation, the best correlation and the data range.

In contrast to the long term mean prediction efficacy, which peaked at about $17 \%$ for $2500 \mathrm{~J} \mathrm{DPE} / \mathrm{m}^{3}$, the best prediction efficacy achieved in any test period was $63 \%$, also at about $2500 \mathrm{~J}$. Despite this seemingly wide variation in efficacy, the general trends for the relations are the same.

If this holds true for subsequent data from other mines a useful relation is defined:

$$
P\left(x>M_{L}\right)=\alpha f\left(D P E, M_{L}\right)
$$

Where $\mathrm{P}\left(\mathrm{x}>\mathrm{M}_{\mathrm{L}}\right)$ is the probability of an event $\mathrm{x}$ exceeding a particular magnitude, $\alpha$ is some scalar that varies between study periods and $\mathrm{M}_{\mathrm{L}}$ is the magnitude. It is a key observation that the function $\mathrm{f}\left(\mathrm{DPE}, \mathrm{M}_{\mathrm{L}}\right)$, which is calculated using the test-block evaluation method in this case, has a similar shape, maximum and minimum over time.

This consistency of the peak and decay for event probability relative to DPE are to be expected, as these would certainly be properties of the rock mass.

More interesting is that the ratio of the probabilities of events at different magnitudes is roughly constant for most of the DPE range. For example at DPE $=2500 \mathrm{~J} / \mathrm{m}^{3}, \mathrm{P}$ (any event) $=17 \%$ is about twice $\mathrm{P}\left(-0.4 \mathrm{M}_{\mathrm{L}}\right)=$ $9 \%$ and more than 5 times $\mathrm{P}\left(0 \mathrm{M}_{\mathrm{L}}\right)=3 \%$, for both the long term average correlation and the best correlation periods in the study. The ratios are very similar at non-peak levels of DPE as well. 


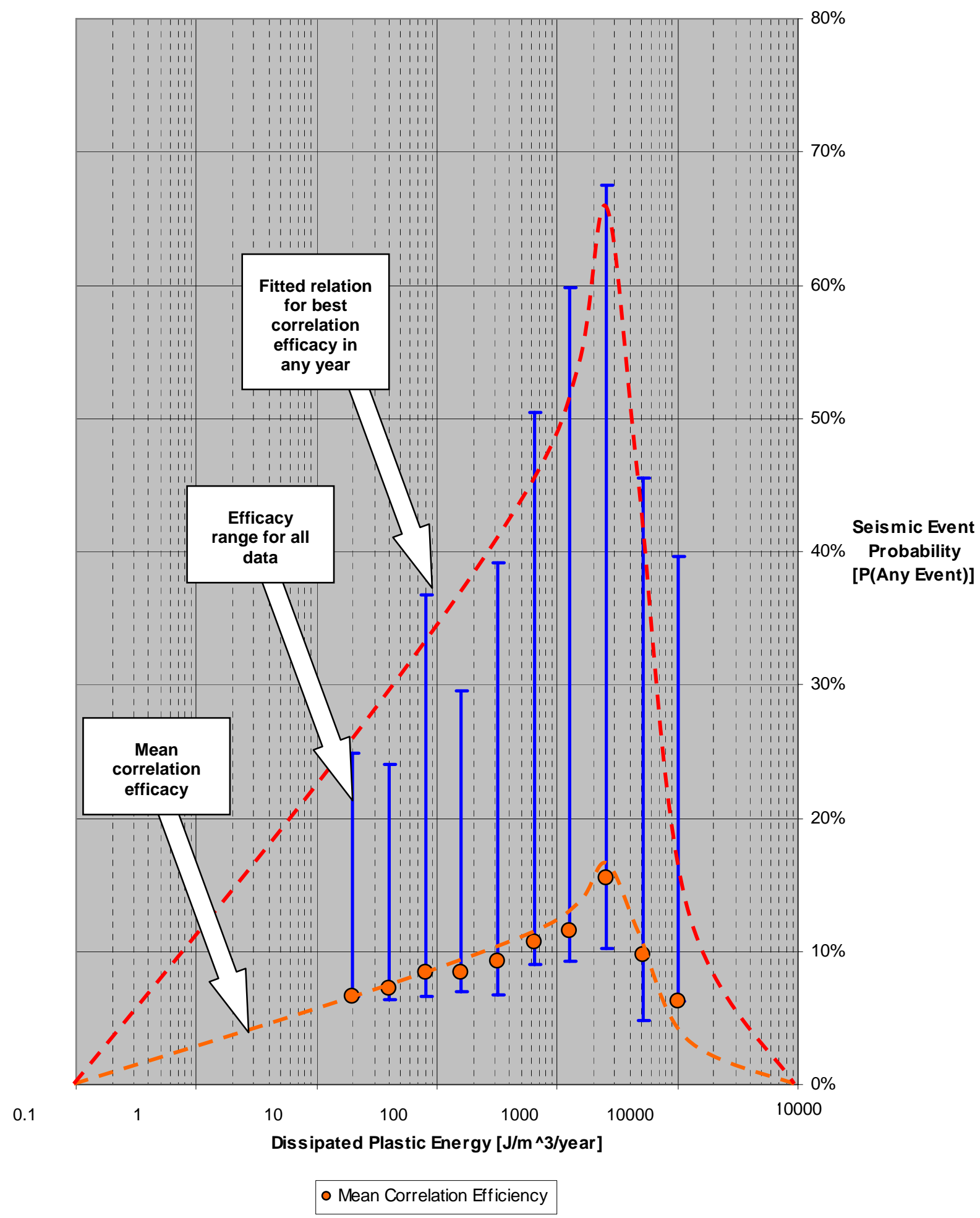

Figure 4 Probability of a seismic event within $20 \mathrm{~m}$ at any magnitude as a function of modelled DPE 


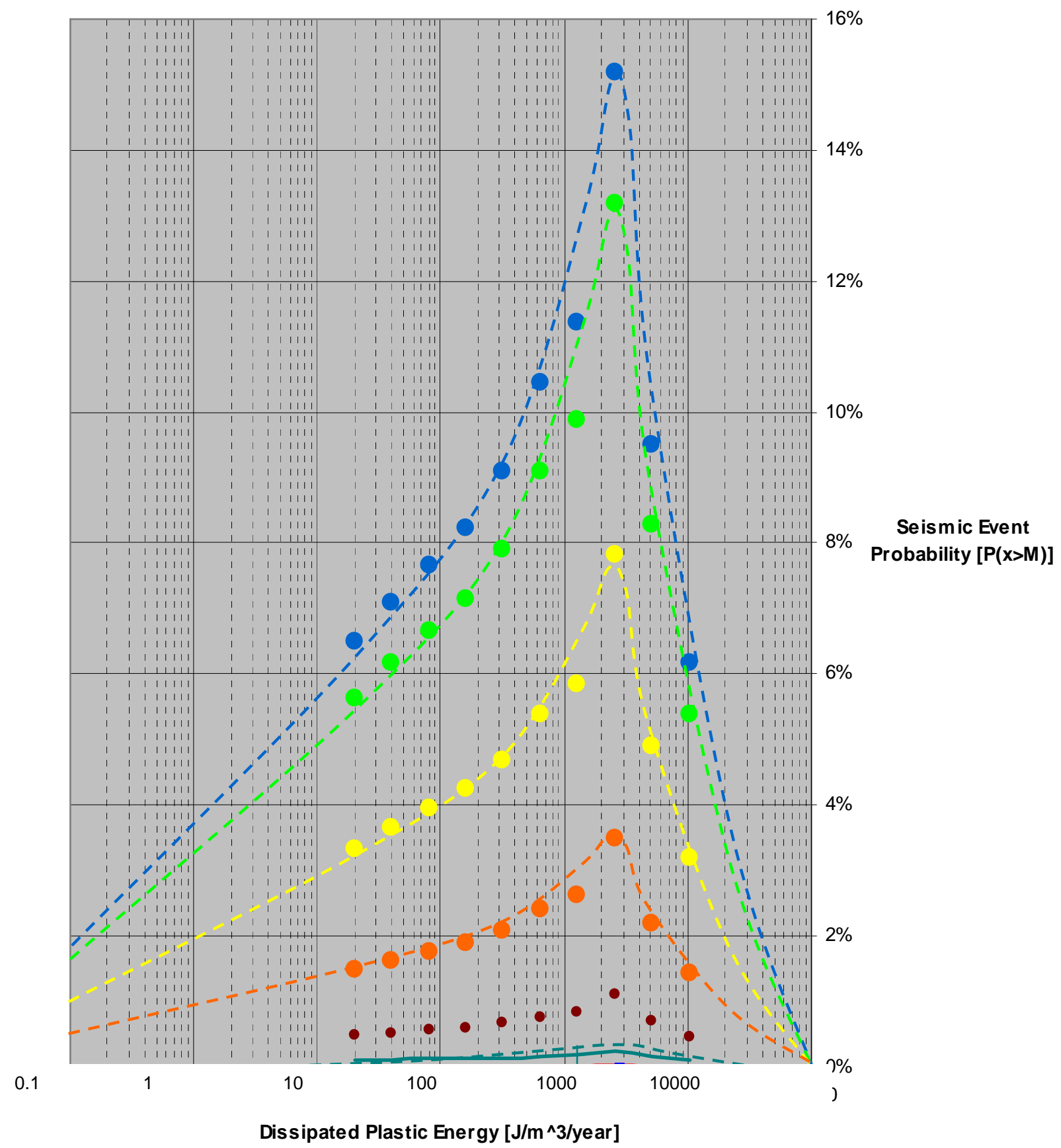

$-1.6 \bullet-1.2 \bullet \bullet-0.8 \bullet \begin{array}{ccccccc}\bullet & \bullet .4 & \bullet & \bullet & 0.4 & \longrightarrow & \longrightarrow \\ -1.6\end{array}$

Figure 5 Probability of a seismic event within $20 \mathrm{~m}$ exceeding listed magnitudes, as a function of modelled DPE

In effect, this ratio can be described:

$$
\frac{P\left(x>M_{L}\right)}{P(x>-\infty)}=f\left(M_{L}\right)
$$

If one recalls from the Gutenberg-Richter relation that the relative proportions of event occurring within magnitude ranges and magnitude is constant over time for much of the seismic population, then this constancy in the relative proportion of events of different magnitudes would be expected, and it may 
therefore be possible that the function describing $\mathrm{P}\left(\mathrm{x}>\mathrm{M}_{\mathrm{L}}\right)$ and the Gutenberg-Richter relation are linearly related or the same.

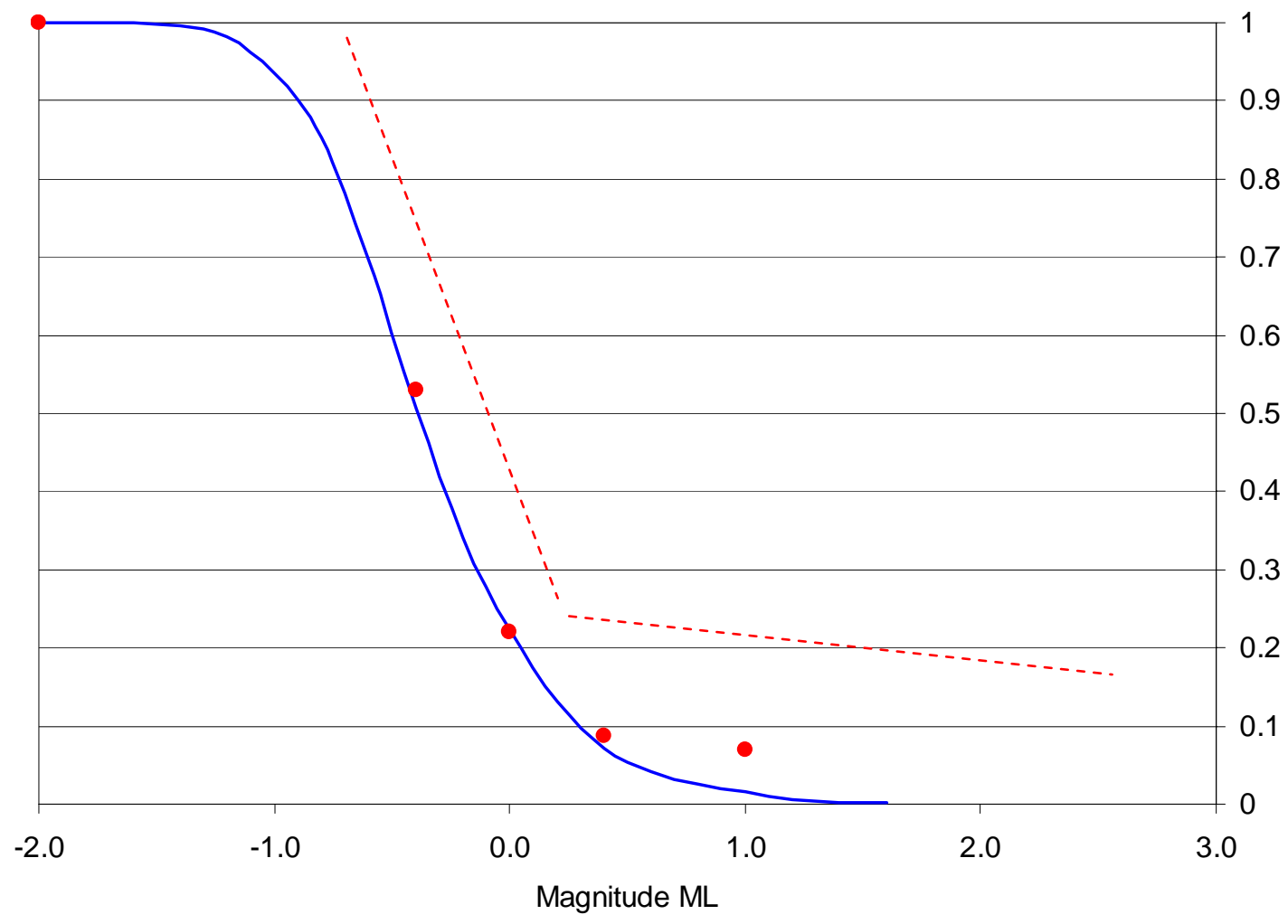

1

\# of Events $>M$ / Total Events : Measured seismic population

- $\mathrm{P}(\mathrm{x}>\mathrm{M}) / \mathrm{P}$ (any event) : Inferred by Modelled DPE

\section{Figure 6 A comparison of the relative occurrence of events at given magnitudes based on the observed Gutenberg Richter Relation, and the predicted by the long term mean prediction efficacy}

To test this hypothesis, equation 3 for the mean efficacies from the model is plotted against the modelled ratio of $\mathrm{P}\left(\mathrm{x}>\mathrm{M}_{\mathrm{L}}\right)$ to $\mathrm{P}(\mathrm{x}>-\infty)$ in Figure 6 . It is a direct comparison of the relative occurrence of events at given magnitudes based on the actual observed Gutenberg Richter relation, and that predicted by the long term mean prediction efficacy for DPE.

This effectively compares the ability of the model to predict event occurrence for different magnitudes and the ratio that occurs in practice, and the correspondence between the measured and modelled data is excellent for magnitudes up to $0.3 \mathrm{M}_{\mathrm{L}}$. The over-prediction of events with magnitudes greater than about $0.3 \mathrm{M}_{\mathrm{L}}$ could be due to the statistical influence of so few events at that magnitude, incompatibility between model step size or representative length scales and the size of such large events, or else it may be a consequence of rarity; such events may occur due to factors not statistically sampled by the rest of the seismic population or the timing of such events may be more random. Research into this high-magnitude event disparity is continuing, but there are some early indications the magnitude of the disparity correlates with large event potential.

The remaining variability in the relation between DPE and event probability is $\alpha$, which is the scalar in equation 3 describing the variability in prediction efficacy year to year for the representative sections of the curve. As discussed, the shape of the function in equation 3 appears to be a constant for the rock mass, so the most likely influence on $\alpha$ is the intensity of DPE release, namely the proportion of the seismogenic volume which contains high levels of DPE rate in a time period. 


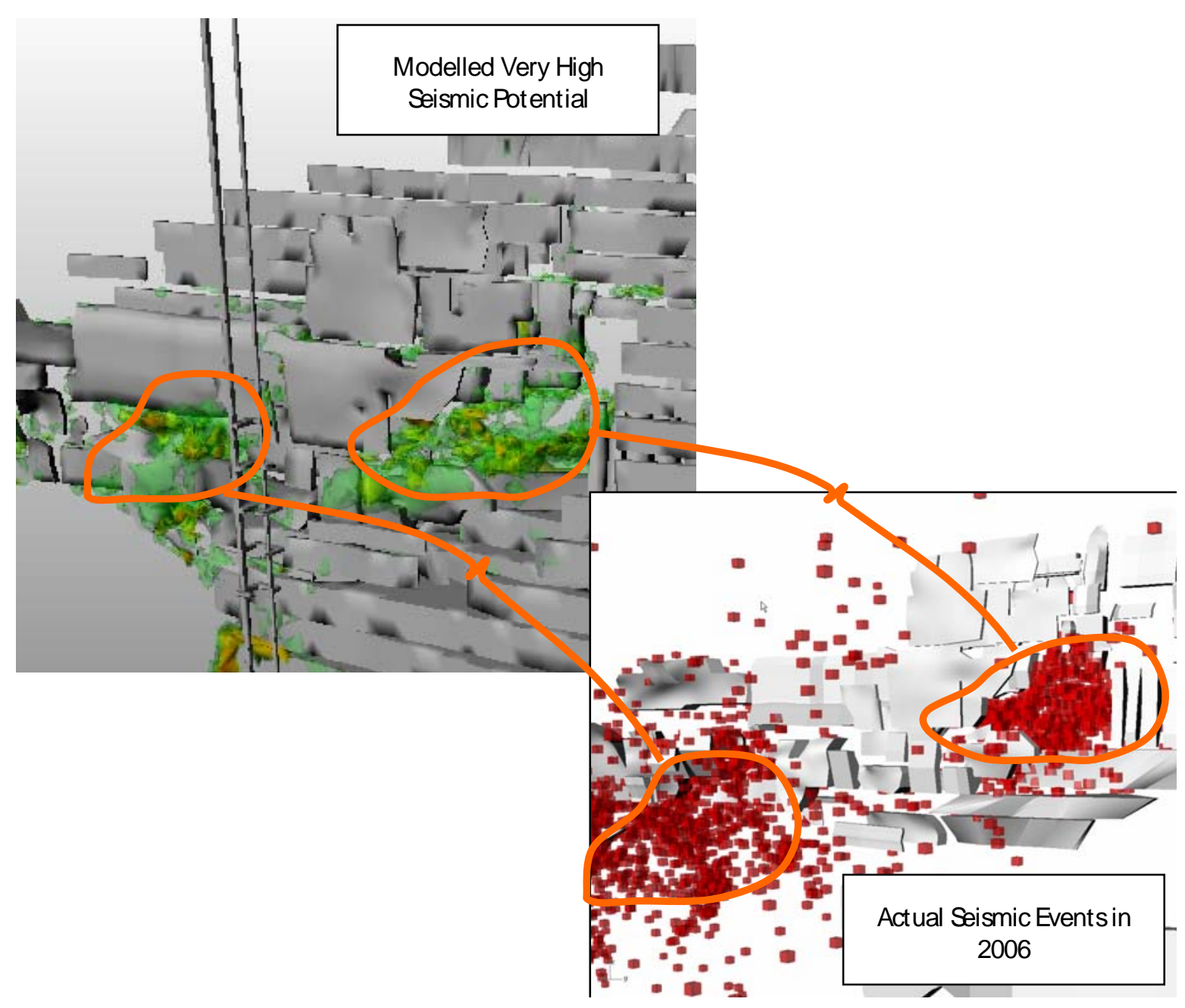

Figure 7 A comparison of spatial clustering of seismicity at the mine for 1 year and DPE contours

Anecdotally it is well known that rapid changes in the damage volume in a rock mass will correspond to higher likelihood of large seismic events, so it is possible that the main control on $\alpha$ may simply be whether or not there are large closed volumes of intense DPE release in any test period. Equation 3 confirms that it isn't the actual level of DPE that influences prediction efficacy directly, and that there is another underlying variable, $\alpha$ influencing peak seismic activity.

In effect, $\alpha$ may describe the influence of the size and shape of the DPE volume in a unit of time on how damage will nucleate and coalesce as individual seismic events, or hypothetically:

$$
\alpha=f(M)
$$

Where $\mathrm{M}$ is the modelled moment (scalars of the modelled moment tensor) of a closed volume of DPE. Again, this hypothesis is the subject of ongoing research.

Whatever the controls on the variation of $\alpha$, it is apparent that there are additional variables that need to be considered to further improve prediction of event probability, but it is clear that the maximum previously observed event probability and a mean event probability can be described by DPE for the majority of the magnitude ranges. At this stage, it would be recommended that the mean and highest indicated event probability be used to describe planning ranges for seismic risk.

This use of a range of probabilities is not an onerous imposition on planning as the prediction efficacies for the mean event probability are still extremely high compared to traditional measures of seismic potential such as ERR, ESS or seismic potency. 
It can also be concluded based on the observed fit between model and actual results that the model is very well calibrated. It simply wouldn't be possible to get such a good match between the onset of seismicity measured by zero DPE, the consistency of the peak seismic efficacy and the close match for predicted and actual event populations if the calibration was not very good.

In Figure 7 an example of the good match between DPE and seismic event occurrence is shown for a period where the model data has been used as a predictor. The clustering of seismicity closely follows the contours of DPE.

\subsection{Mine 3: Primary and secondary stopes, 4 month study using portable seismic system}

Mine 3 is a unique case study, because the available seismic data spanned just a few months, and was collected using two portable ESG seismic systems. The model of the mine was calibrated using observations of rock damage in development. The DPE correlation is shown in Figure 8.

Owing to the simple nature of the seismic system, only about $80 \%$ of the data could be used with the remainder of events rejected owing to poor location accuracy. Most of the rejected data was located outside the seismic array, so it is reasonable not to include it in the analysis. The remaining volume of data was very small, resulting in some practical uncertainty in the application of the result. The curves on the graph other than the 'any event' curve were also calculated using the relation described in equations 3 and 4 , because there simply too few events to calculate a DPE relation at each magnitude range.

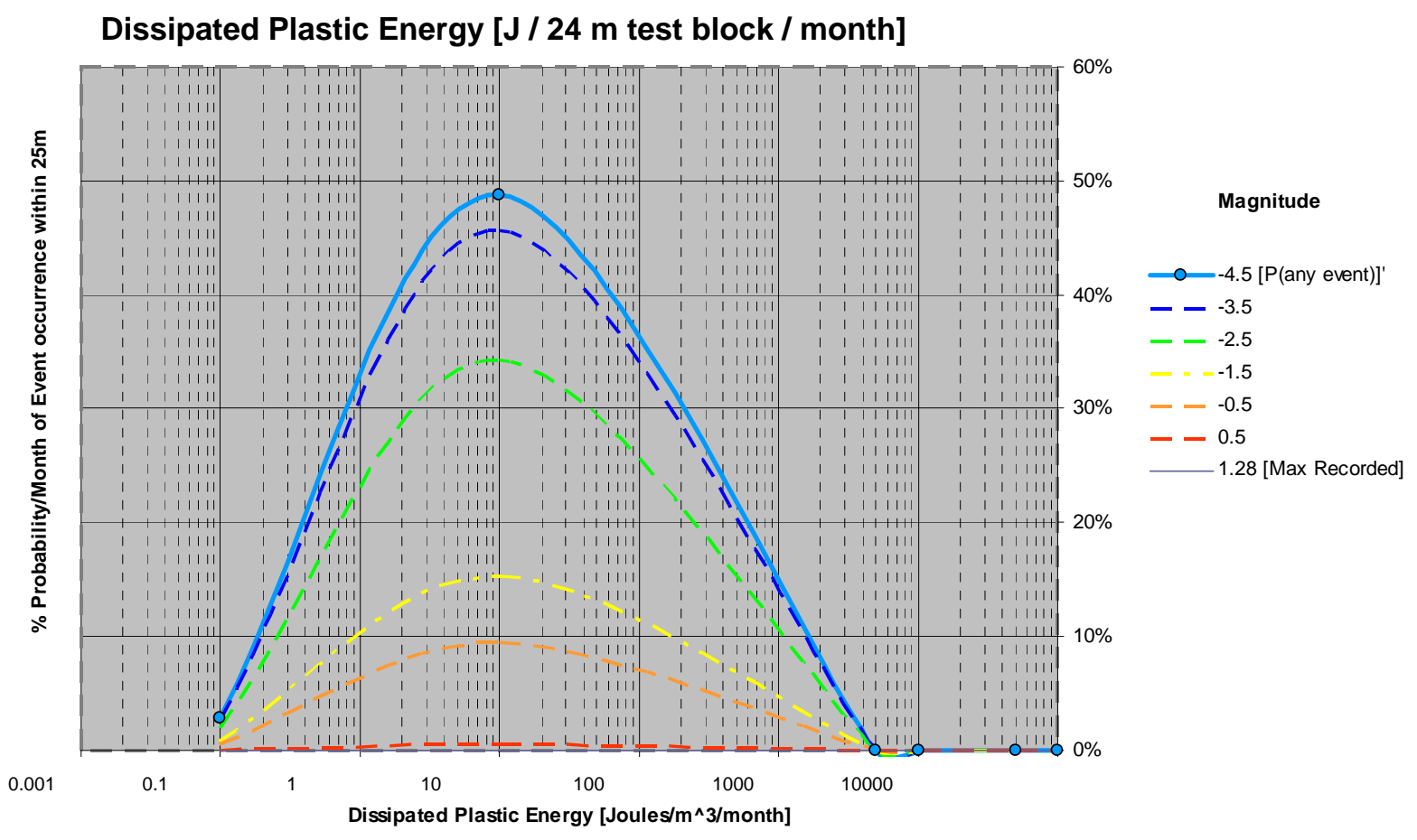

Figure 8 Probability of a seismic event within $24 \mathbf{m}$ at any magnitude as a function of modelled DPE

Excluding the effects of this approximation, the nature of the relation between DPE and seismicity replicates the observations from Mines 1 and 2.

1. The graph allows the modelled DPE to be interpreted as event probability directly with a quantifiable error range.

2. The graphs show there is near-zero event probability at zero DPE release rate.

3. There is a peak DPE beyond which the event probability begins to decrease.

The value of this study is that it shows early measurement of seismicity may give a very good indication of longer term rock mass response. 


\section{Conclusions}

At 2 mines where sufficient data was available, analysis showed that there is a useable correlation between DPE and seismic event probability. At a third mine with limited data, the nature of the relation was observed. Despite the short measurement period and small population of seismic events, the simplest interpretation of the result suggests it is reasonable to expect that over time, a useable, quantitative relation will be developed and that modelling of the earliest measurements of seismicity will be worthwhile.

The correlation observed at all three mines allows a quantitative estimate of the probability of having a seismic event within a certain distance of properly conditioned areas within a 3D- non-linear FE model and an estimate of the probability of the event exceeding a certain magnitude based on the previously measured Gutenberg Richter relation.

This is a useful result. In time, with more data it will be possible to improve the resolution of the prediction and our understanding of nucleation processes of seismic event.

\section{References}

Beck, D., Reusch, F., Arndt, S., Thin, I., Heap, M., Tyler, B. and Stone, C. (2006) Numerical modelling of seismogenic development during cave initiation, propagation and breakthrough. Proceedings $3^{\text {rd }}$ International Seminar on Deep and High Stress Mining, Quebec, Canada, 2006.

Beck, D.A. and Brady, B.H.G. (2001) A numerical method for engineering management of induced seismic risk in hard rock nining. Proceedings $5^{\text {th }}$ International Symposium on Rockbursts and Seismicity in Mines, RaSiM5, SAIMM, South Africa, G. van Aswegen, R.J. Durrheim, W.D. Ortlepp (eds), pp. 457-463. 